\title{
First Beam Circulation Test of an Electron Storage/Stretcher Ring, KSR
}

\author{
T.Shirai, T.Sugimura, Y.Iwashita, H.Fujita, H.Tonguu, A.Noda, M.Inoue \\ NSRF, Institute for Chemical Research, Kyoto University \\ Gokasho, Uji, Kyoto 611-0011, Japan
}

\begin{abstract}
In Kyoto University, we are constructing a compact electron storage/stretcher ring (KSR). The circumference is $25.7 \mathrm{~m}$ and the maximum beam energy is $300 \mathrm{MeV}$. The electron beam is injected from a $100 \mathrm{MeV}$ linac through the doubly achromatic transport line. The beam test of the injection line was finished and the beam size and the dispersion were measured. The results are consistent with the design calculation. The beam current is $100 \mathrm{~mA}$ and the energy spread is $+/-0.5 \%$ (FWHM). KSR uses the 1-turn injection scheme. The bump orbit is created by the perturbation magnet for the injection. The test of the bump magnet was carried out.
\end{abstract}

\section{INTRODUCTION}

A compact electron ring (Kaken Storage Ring, KSR) is now under construction at the Institute for Chemical Research, Kyoto University [1]. The main parameters are shown in table 1 and the view of the ring is shown in Fig.1. KSR has a triple bend doubly achromatic lattice (TBDA). It has two operation modes. One is a stretcher mode. The $100 \mathrm{MeV}$ electron beam is injected from an Sband electron linac [2] and extracted slowly using the $3^{\text {rd }}$ order resonance and an RF-knockout method [3]. Experiments of the coherent $\mathrm{X}$-ray radiation are planned using the extracted beam. The another one is a storage mode. The injection energy is $100 \mathrm{MeV}$ and it is accelerated up to $300 \mathrm{MeV}$. The design beam current is $100 \mathrm{~mA}$ and the critical wavelength is $17 \mathrm{~nm}$. In the storage mode, some experiments are prepared using the insertion devices in the long straight section.

The injector linac consists of a thermal electron gun, a buncher and three accelerator tubes. The output beam energy is $100 \mathrm{MeV}$. The maximum beam pulse width is 1 $\mu \mathrm{sec}$ and the repetition is $20 \mathrm{~Hz}$. For the injection to KSR, the pulse width is $0.1 \mu \mathrm{sec}$, which correspond to the revolution time of KSR.

\section{INJECTION LINE}

\subsection{Design}

The injection and the extraction line for KSR are shown in Fig. 2 (a). There are two bending magnets, two doublets of the quadrupole magnets and the inflector septum magnet in the injection line. The design goal of the injection line is to satisfy the following three
Table 1 Main parameters of KSR

\begin{tabular}{ll}
\hline Circumference & $25.689 \mathrm{~m}$ \\
Lattice & TBDA \\
Curvature & $0.835 \mathrm{~m}$ \\
Length of straight section & $5.619 \mathrm{~m}$ \\
Tune & $(2.36,1.43)$ \\
RF frequency & $116.7 \mathrm{MHz}$ \\
\hline Stretcher mode & \\
$\quad$ Beam energy & $100 \mathrm{MeV}$ \\
Extracted beam (design) & $5 \times 10^{10} \mathrm{e}^{-} / \mathrm{sec}$ \\
$\quad$ Repetition & $1 \mathrm{~Hz}$ \\
\hline Storage mode & \\
$\quad$ Maximum energy & $300 \mathrm{MeV}$ \\
$\quad$ Stored current (design) & $100 \mathrm{~mA}$ \\
Critical wavelength & $17 \mathrm{~nm}$ \\
\hline
\end{tabular}

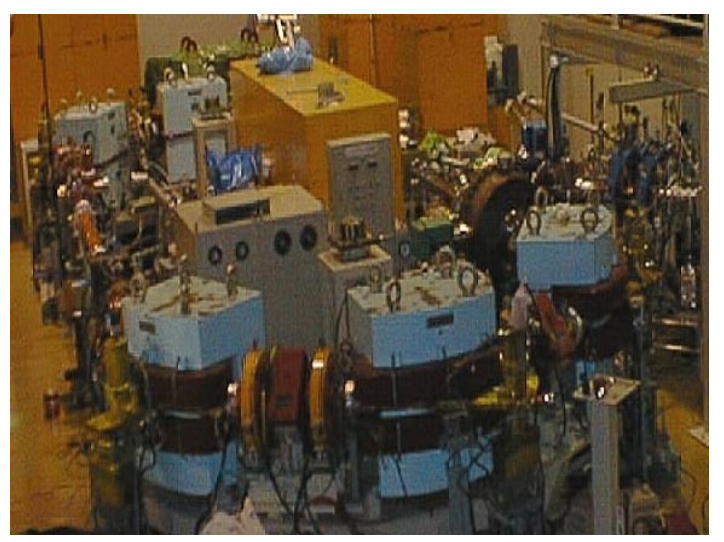

Figure 1 View of KSR. The injection line exists in the right side.

conditions at the injection point,

$\mathrm{I}: \mathrm{D}_{\mathrm{x}}=\mathrm{D}_{\mathrm{px}}=0$ (doubly achromatic condition),

II : $\beta_{y}=6.3, \alpha_{y}=-2.3$ (matching condition in Y-Y' ),

III : $\beta_{x}<7$ (aperture limit of the septum).

But it is difficult to satisfy all conditions because the degree of freedom of the magnet arrangement is limited due to the small area around the linac and KSR. We adopt the large edge of $40^{\circ}$ on the exit pole face of BM1 to satisfy the doubly achromatic condition. 


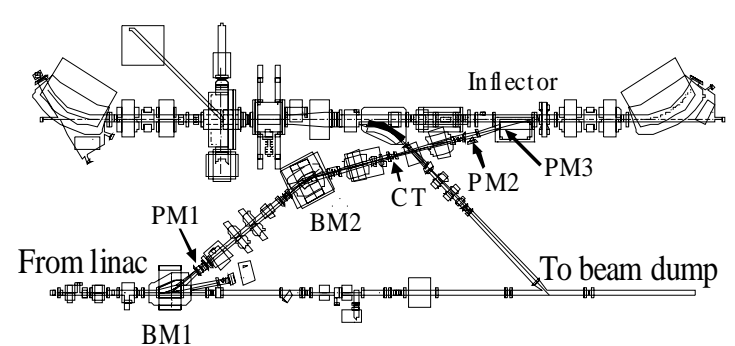

Figure 2 (a) Injection line from the linac to KSR.

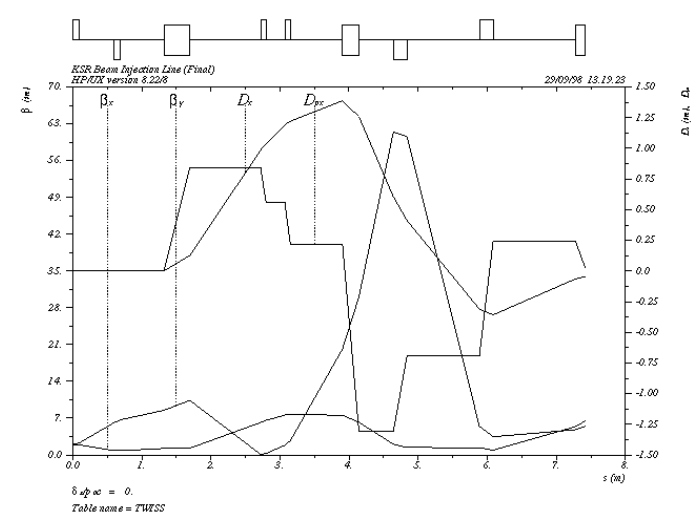

Figure 2 (b) Twiss parameters in the injection line.

The beta function $\left(\beta_{\mathrm{x}}, \beta_{\mathrm{y}}\right)$, the dispersion function

$\left(D_{x}\right)$ and its derivative $\left(D_{n x}\right)$ are shown.

Table 2 Twiss parameters at the injection point of KSR.

\begin{tabular}{lll}
\hline & Simulation results & KSR acceptance \\
\hline$\beta_{\mathrm{x}}(\mathrm{m})$ & 6.5 & 6.4 \\
$\alpha_{\mathrm{x}}$ & -2.5 & -0.43 \\
$\beta_{\mathrm{y}}(\mathrm{m})$ & 5.4 & 6.23 \\
$\alpha_{\mathrm{y}}$ & -2.6 & -2.3 \\
$\mathrm{D}_{\mathrm{x}}(\mathrm{m})$ & 0 & 0 \\
$\mathrm{D}_{\mathrm{px}}$ & 0 & 0 \\
\hline
\end{tabular}

Figure 2 (b) shows the beta and the dispersion function in the injection line. It is calculated by the program code, MAD [4]. The initial point of the simulation is at the entrance of the quadrupole magnet behind the accelerator tube No.3, where $\beta_{\mathrm{x}}=3.0 \mathrm{~m}, \alpha_{\mathrm{x}}=2.0, \beta_{\mathrm{y}}=3.0 \mathrm{~m}, \alpha_{\mathrm{y}}=$ 2.0. The Twiss parameters at the injection point are shown in table 2. The dispersion and its derivative are zero. There is a mismatching in Y-Y' plane. Figure 3 shows a ring acceptance and the beam emittance at the injection point. The beam emittance is assumed to be 0.4 $\pi$ 'mmmrad and $1.3 \pi \mathrm{mm}^{\prime} \mathrm{mrad}$ in $\mathrm{X}-\mathrm{X}^{\prime}$ and $\mathrm{Y}^{\prime} \mathrm{Y}^{\prime}$ phase space, respectively [5]. The acceptances are 161 $\pi \mathrm{mm}^{\prime} \mathrm{mrad}$ and $11.7 \pi \mathrm{mm}$ mrad in the $\mathrm{X}-\mathrm{X}^{\prime}$ and $\mathrm{Y}^{\prime} \mathrm{Y}^{\prime}$ planes. The beam ellipse is located inside the acceptance but the emittance becomes 2.1 times larger due to the mismatching in Y-Y' phase space.

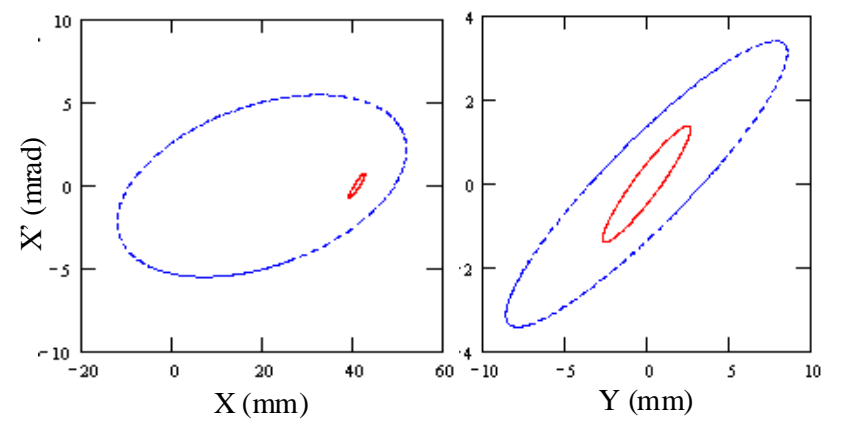

Figure 3 Beam emittance (solid line) and the ring acceptance (dotted line) at the injection point. The beam emittance is $0.4 \pi \mathrm{mm} m$ rad and $1.3 \pi \mathrm{mm} m$ rad in the $\mathrm{X}$ $X^{\prime}$ and $Y-Y^{\prime}$ planes, respectively. The acceptances are 161 $\pi \mathrm{mm}$ mrad and $11.7 \pi \mathrm{mm}$ mrad in the $\mathrm{X}-\mathrm{X}^{\prime}$ and $\mathrm{Y}-\mathrm{Y}^{\prime}$ planes.

\subsection{Measurements}

In the injection line, there are 3 beam profile monitors (PM1, PM2 and PM3) and one current transformer (CT). The beam sizes are measured by the profile monitors. The results are almost consistent with the MAD calculation and the beam size at PM3 is $6 \mathrm{~mm}$, which is smaller than the septum aperture $(8 \mathrm{~mm})$. The dispersion is also measured by the profile monitors. The beam energy is changed by the RF phase adjustment of the accelerator tube No.3 and the beam position shift were measured. The measured dispersion at PM2 is $17 \mathrm{~cm}$, where the design value is $28 \mathrm{~cm}$. The energy spread was measured by PM1. It is +/- $0.5 \%$ (FWHM) and +/-1.2\% (total) with the beam current of $100 \mathrm{~mA}$ and the pulse width of 100 nsec. It is acceptable value for the chromaticity correction by sextupole magnets [6].

\section{INJECTION SCHEME IN KSR}

The layout of KSR and the Twiss parameters are shown in figure 3 (a) and (b). The characteristic point of the optics is long dispersion free sections. The injection also uses this section. The injected beam is bent 15 degree by the septum magnet and merged into the ring orbit. The injection point is $41 \mathrm{~mm}$ apart from the centre orbit.

\subsection{Stretcher Mode}

In the stretcher mode, one turn injection is used. The beam pulse width is $100 \mathrm{nsec}$ and the beam current is 100 $\mathrm{mA}$. The repetition is $1 \mathrm{~Hz}$. To inject the beam, the bump orbit is created by a beam perturbation magnet (BPM). Figure 4 shows the waveform of the excitation current for the BPM. The maximum magnetic field of BPM is 360 Gauss and the time constant is $300 \mathrm{nsec}$. Figure 5 shows the beam distribution at the injection point in $X-X^{\prime}$ phase space when the kick angle of the BPM is $6 \mathrm{mrad}$. The index in the figure shows a circulation number of the 
beam. The shaded area is a septum. To improve the emittance of the stored beam, the more precise control of the close orbit by the correction magnets is needed.

\subsection{Storage Mode}

In the storage mode, the same injection scheme is used. The differences are the repetition and a beam window. The beam repetition is $0.2 \mathrm{~Hz}$ because the damping time is $9.6 \mathrm{sec}$ in the horizontal direction. The thin foil is attached in the inflector septum as a beam window to isolate the vacuum system between the beam line and the ring because the design vacuum pressure is $10^{-9}$ Torr in the ring, while it is $10^{-7}$ Torr in the beam line. The foil is Kapton with the thickness of $25 \mu \mathrm{m}$. The emittance growth is induced by the multiple scattering in the foil. The angular distribution $f(\theta)$ of the scattered electron becomes the gaussian function and the width can be estimated by the Moliere's formula [7],

$$
\begin{aligned}
& f(\theta)=A \exp \left(-\frac{\theta^{2}}{\sigma^{2}}\right)=A \exp \left(-\frac{\theta^{2}}{B \chi^{2}}\right), \\
& \chi=\sqrt{4 \pi N\left(Z^{2}+Z\right)} \frac{e^{2}}{p c},
\end{aligned}
$$

where $\mathrm{p}$ is a momentum of the electron. The width $\sigma$ is $0.85 \mathrm{mrad}$ in this case. The emittance becomes 2.0 times larger. This effect leads to the beam loss of $30 \%$. So the multiple injection after the damping is needed in the storage mode.

\section{ACKNOWLEDGEMENTS}

The authors would like to present our thanks to the support of the HIMAC group in NIRS and the accelerator group in JAERI. Special thanks to Mr. Kazama for his technical help.

\section{REFERENCES}

[1] A.Noda, et al., "Electron Storage and Stretcher Ring, KSR", Proc. of the 5th European Particle Accelerator Conference, Sitges (Barcelona), Spain, 451-453 (1996)

[2] T.Shirai, et al., "A $100 \mathrm{MeV}$ Injector for the Electron Storage Ring at Kyoto University", Proc. of the Particle Accelerator Conference, Dallas, USA, 1079 (1995).

[3] A.Noda, et al., "Slow Beam Extraction at KSR with Combination of Third Order Resonance and RFKO", contribution paper in this conference.

[4] H. Grote, F. Chistoph Iselein. The MAD Program. (Methodical Accelerator Design) Version 8.10 User's Reference Manual. CERN, 1993

[5] T. Sugimura, et al., "Present Status of the Electron Linac as the Injector for KSR", Proc. of the Particle Accelerator Conference, Vancouver, Canada, 1206 (1997)

[6] A.Noda, et al., "KSR as a pulse stretcher", Proc. of the Particle Accelerator Conference, Vancouver, Canada, 339 (1997)

[7] A.O.Hanson, et al., "Measurement of Multiple Scattering of 15.7 MeV Electrons", Physical Review 84, 634 (1951)

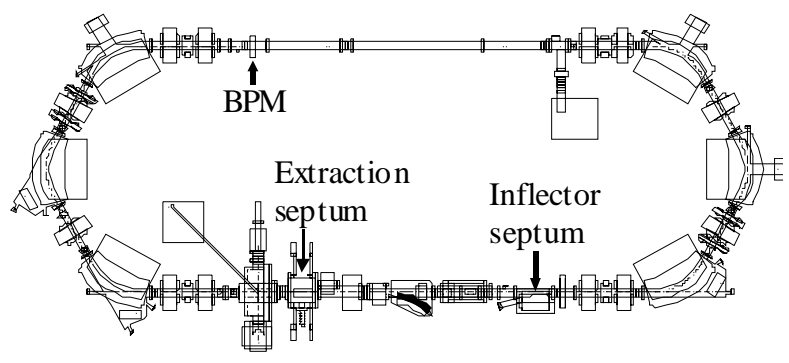

Figure 3 (a) Layout of KSR

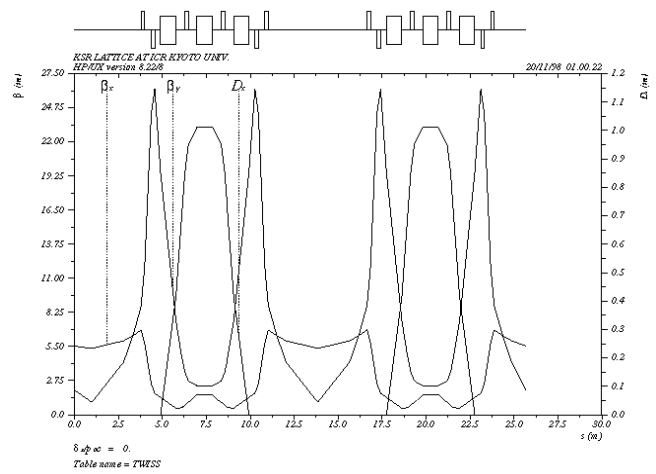

Figure 3 (b) Twiss parameters in KSR.

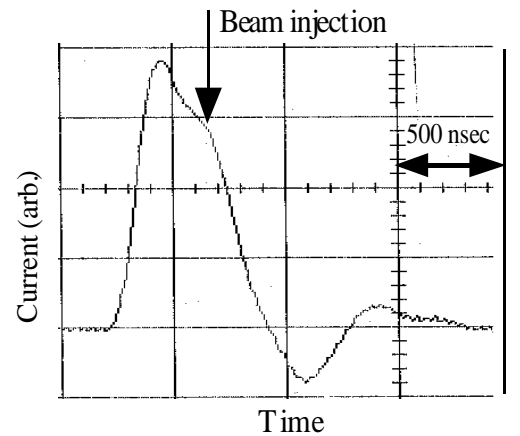

Figure 4 Waveform of the excitation current of the BPM.

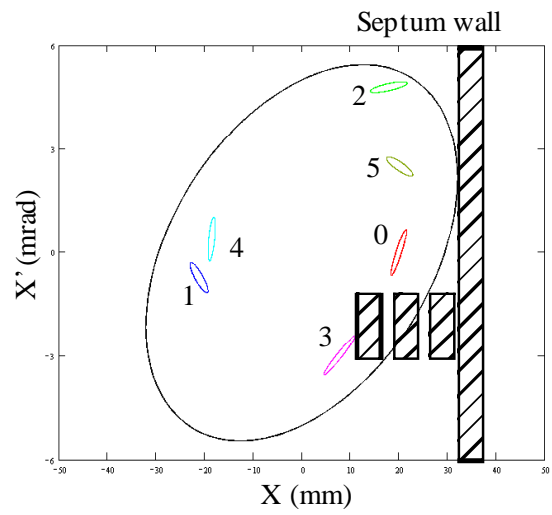

Figure 5 Beam distribution at the injection point in $\mathrm{X}-\mathrm{X}^{\prime}$ phase space when the kick angle by the BPM is $6 \mathrm{mrad}$. The index in the figure shows a circulation number of the beam. The shaded area is a septum. 